\title{
The Application Research of Geometric Language in Modern Furniture
}

\author{
Anlin Huang ${ }^{1,2}$, Jianhua Lyu ${ }^{1,2, *}$ and Ming Chen ${ }^{1,2}$ \\ ${ }^{1}$ College of Forestry, Sichuan Agricultural University, Chengdu, Sichuan, 611130, China \\ ${ }^{2}$ Key Laboratory of Wood Industry and Furniture Engineering, Chengdu, Sichuan, 611130, China
}

\begin{abstract}
From the perspective of modern furniture innovative design, this paper analyzes the application extent of geometric language in modern furniture, and provides new design ideas for modern furniture symbolization. By studying the elements of geometric language, such as point, line, surface, color block, shape, through combination, division, superposition, reconstruction and other methods, a variety of symbolic modelling elements are created. Finally, three principles of product symbolic design are summarized, and at the same time, combined with color matching, material selection, man-machine size, using functions, which could be applied into modern furniture design.
\end{abstract}

\section{Introduction}

The improvement of people's taste in furniture relies on the continuous improvement of people's living standards and the rapid development of science and technology, and people's requirements for furniture are not only to meet the use function, but also to gradually pay attention to the appearance and connotation of furniture. Therefore, it is combined with the aesthetic of modern people on the basis of keeping the simplicity, functionality and coordination with the interior space of modern furniture, through in-depth analysis of the development trend of modern furniture design, the application of geometric language in modern furniture and furniture symbolic design. The simple form of furniture is combined with the abstract geometric language, which makes the shape of furniture more novel, natural, flexible and gives furniture new connotation. Different geometric languages create a variety of unique shapes, which brings continuous inspiration to designers to better meet the needs of customers, to let customers experience the different beauty brought by different shapes, and to promote the development of furniture design [1].

\section{Geometric language}

\subsection{A sketch of geometric language}

Geometric language is a kind of composition art which is composed of points, lines, surfaces, color blocks and shapes. As early as ancient classical times, ancient Greeks began to study it. Through the symbolization of concrete objective image, it is abstracted into geometric form with simple structure, clear lines and rich connotation

The development of geometric language has also experienced several periods including germination, maturity and diversification. Geometric language has entered a period of diversified development, for the acceptance of abstract visual expression and the improvement of people's aesthetic level. Different geometric languages give people different psychological feelings, and they have different degrees of application in painting, graphic design, clothing design, interior design, furniture design and so on.

\subsection{The basic constitutive elements of geometric language}

\subsubsection{Point}

In a geometric sense, a point represents only the position, neither the direction nor the size. But in the sense of artistic modeling, points not only have the difference of size and shape, but also express different emotions. Points have the function of emphasizing for its appearance of round and full; square points are stable and solid; triangle points are sharp and stable; irregular points are free and flexible. The different arrangement and combination of points will also create the concave and convex, static, dynamic and other varied pictures, which can make the furniture form more varied and vivid when reasonably applied to modern furniture design.

\subsubsection{Line}

Line has strong emotional characteristics, such as, straight line is simple and rigorous, but curve is gentle 
and soft. The use of line form can be seen everywhere in furniture design, such as the overall form, structure, decorative pattern, and so on. Different furniture shapes are formed for different thickness, length, straightness, arrangement and combination of lines form providing more possibilities for furniture appearance design. The System Table in Figure 1 is a set of three-dimensional objects made of tubular stainless steel formed by surface, which is composed of lines. In the overall modeling design, the lines in horizontal and vertical formats are used, for the horizontal lines are stable, and the vertical lines are directly clear; when comes to the shapes and forms, the most basic unmodified geometric forms are used.

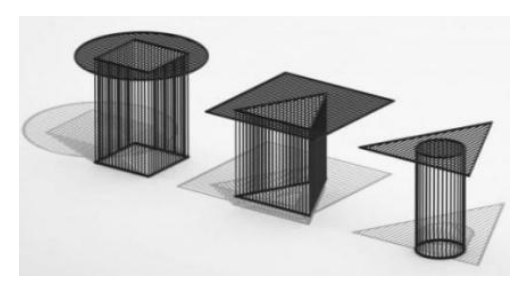

Figure 1 System Table

\subsection{An analysis of the application of geometric language in modern furniture}

From the perspective of the development of furniture in the world, the design of modern furniture is developing in the direction of advanced technology, feasible production, reasonable economy, beautiful style and safe use [3]. The application of geometric language in modern furniture mainly includes two aspects: the independent use of geometric language and the combination of geometric languages. Geometric language, with its simple shape, smooth lines and rich connotation, occupies an important position in modern furniture design. However, in the process of practical application, there are some problems such as unclear features, improper combination, lacking of order and inadequate handling of details.

Charles Rainey Mackintosh, a famous British designer, uses geometric design language to express his style, which is simple and has the sense of highly decorative taste. His design does not have complicated concrete decoration, but, through the abstraction, abstraction and generalization of the objective image to get the design inspiration, which is applied to the furniture design. His "high back chair" is one of his representatives. The use of geometric language in foreign countries has reached a more mature stage, but it is still in the exploratory stage in China. We should open our minds to adapt to the development of the times, and use geometric language to achieve the appearance design innovation of modern furniture.

\subsubsection{Surface}

Surface is an important component of furniture modeling. All the boards are in the shape of surface, only with which furniture can have the function of using and form the shape [2]. The surface has a sense of fullness and stability. The straight surface is more rational for simple and decent, and the curve surface is soft and flexible. The surface can also be formed by the arrangement and combination of points and lines. In Figure 2, CHEROKEE series designed by Daria Zinovatnaya, a Ukrainian designer, the side cabinet body of which is a geometric curved surface, and the panel is divided into three circular surfaces by several different colors, which is vivid, relaxed and round.

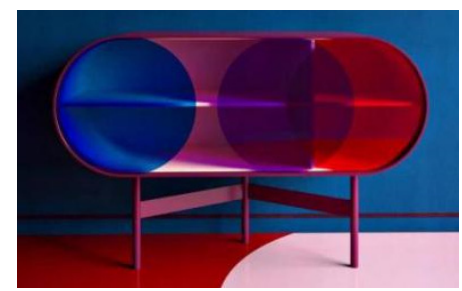

Figure 2 CHEROKEE

\section{Geometric language and symbolic design of modern furniture}

\subsection{Symbolic design of modern furniture}

Symbolic language includes four types: geometric symbols, literal symbols, symbolic symbols and biological symbols. The symbolic design of modern furniture conveys the cultural connotation of design through geometric language, endows the era characteristics of modern furniture, emphasizes peopleoriented, enhances the sense of user experience, and creates modern furniture products that meet the emotional and human needs of users. The three principles of symbolic design are respectively design following emotion, design creating experience and design following culture.

\subsection{The embodiment of geometric language in three design principles}

\subsubsection{The principle of geometric language follows emotion}

Modern furniture should meet the physiological and psychological needs of consumers at the same time. The focus of furniture design has shifted from satisfying function and practicality to aesthetics, culture, symbol, personality, health and innovation, and geometric language is applied to modern furniture, which causes emotional resonance of users through its unique connotation.

The extraction of geometric language is to refine and generalize the objective things after careful selection, 
transform the concrete things into abstract geometric language, and finally apply it to modern furniture design. From this, we can see that we can extract elements from the concrete forms in nature. There are many objective objects in nature, such as mountains, rivers, sun, moon, stars, animals and plants. These objective objects can provide continuous inspiration for the extraction of geometric language, and also can cause emotional resonance of users. In the process of geometric language extraction, it is not only a simple simplification and generalization, but also an analysis of the shape and structure of the objective image and a simplification and dismantling of the symbols, from the concrete to the abstract, from the clear to the fuzzy, to retain its most symbolic modeling features. Then through the combination, division, superposition, reconstruction and other methods to achieve the purpose of shape like, spirit like and vivid, get the most aesthetic geometric modeling elements, and bring unique emotional feelings to customers.

\subsubsection{The principle of Geometric language creates experience}

Modern furniture products make people get feeling through vision, smell, taste, hearing, touch and other senses. The design should not only meet the usability and ease of use of modern furniture, but also consider giving people a new use experience.

Geometric language can be expressed through color and material differentiation, giving people different visual and tactile experiences. First of all, in terms of color, furniture color matching should follow the basic principles of color matching, grasp the overall color of furniture, and give different colors to points, lines and surfaces through the contrast of color phase, lightness and purity, emphasizing the shape and connotation of geometric language. Geometric language is usually extracted from natural images, and the color matching of modern furniture can be extracted from natural scenery, which can be combined to produce more natural and flexible design elements. Secondly, in terms of materials, the design and application of furniture materials directly determine the aesthetic taste and quality of furniture. The expressive force of furniture materials mainly includes three parts: color expressiveness, shape expressiveness and texture expressiveness [4]. The stone is rough and bulky, the glass is crystal clear, and the metal is cold and hard. Through the separation between different materials, the geometric language can be expressed. Different material combinations give new vitality to the geometric language and give people a new experience. The collocation of color and material transforms the simple form of geometric language into richer and complete design elements.

In terms of function, in the process of applying geometric language to modern furniture, we should consider the size and proportion of points, lines and surfaces. First of all, comply with ergonomics, meet the needs of modern people for furniture function, and have comfort and practicability; second, we should consider the requirements of modern living space for furniture size; at the same time, we should integrate humanoriented design concept into it, so as to achieve harmony among human and furniture production and environment.

\subsubsection{The principle of Geometric language follows cultural}

Designers often get design inspiration by abstracting and generalizing the geometry of objective objects, and then use them independently or in combination to fully express the cultural connotation and modeling implication of geometric language. The different combinations of geometric language also reflect people's ideological and cultural, religious beliefs, aesthetic concepts and life interests, and so on. At the same time, they also have distinctive characteristics of the times and show the spirit of the times.

\section{The application of geometric language in modern furniture design}

\subsection{Geometric language demand in modern furniture}

The geometric language is applied to modern furniture design. The geometric language is obtained by abstracting and generalizing the objective image, and then it is combined, split, superposed and reconstructed. Finally, it is applied to modern furniture design from different angles, forces and directions to realize the geometric of modern furniture and make the form of furniture products more natural, friendly, flexible and novel and the color is more diversified, richer and harmonious. Figure 3 is the BEIKE chair designed by JIHE STUDIO. Its design inspiration comes from the shell in the ocean, the texture of which is extracted, refined and summarized into lines of accumulation and arrangement. Finally, it is applied to the back of the chair, around the sitting surface, reflecting the shell's wrapping sense of the interior, and also expresses a delicate feeling to people.

\subsection{Geometric language design in modern furniture}

The whole process of furniture design is a process of visualizing and symbolizing of creativity and design. The designer selects, transforms and combines the visual elements according to the design intention, and organically associates and codes the visual elements to form a specific symbol system [5]. Geometric language occupies an irreplaceable position in this characteristic symbol system. In Figure 4 is the modern furniture "hand can pick stars" series furniture in study, which is inspired by "sun, moon and stars". In today's era full of fashion and personality, everyone wants to have their own fashion and personality, many of them are full of curiosity, yearning and love for the starry sky, so this modern furniture series wants to give users the feeling of 
starry dream and freedom. This set of furniture selects the shape of "sun, moon and stars" for reconstruction and combination, and applies all the geometric language obtained to the handle, cabinet door and other positions of the furniture. With blue and white as the main colors, and black, green and gold as the embellishment colors,

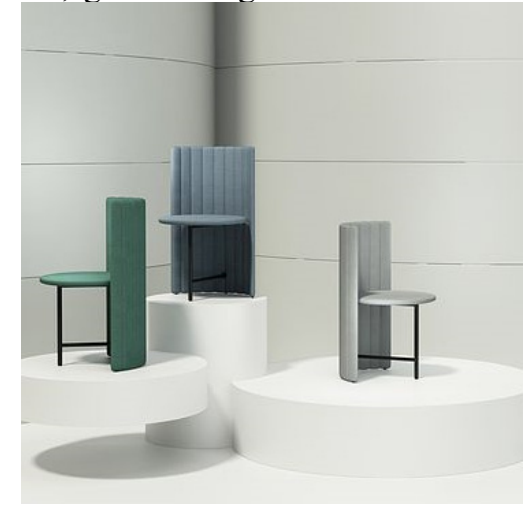

Figure 3 BEIKE

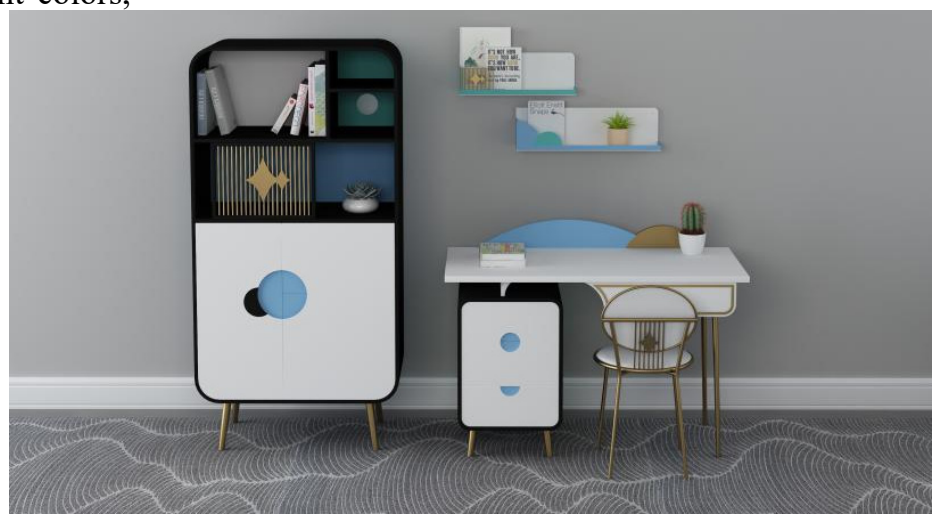

Figure 4 Hand Can Pick Stars

\section{Conclusion}

Under the guidance of the spirit of the times, the trend of modern furniture design is no longer satisfied with the realization of its functions, but has developed into a modern furniture design that needs to be more beautiful, cultural, unique and personalized, and at the same time needs to be more adaptable to the lifestyle, market needs and consumer groups. Therefore, we use symbolic design to provide design inspiration for modern furniture, try to apply geometric language to modern furniture design, improve the cultural connotation and ideological connotation of modern furniture products to better show the spirit of the times, and bring personality and quality to our life.

\section{References}

1. Guan Yufeng, $\mathrm{Xu}$ Gefei. (2016) On the application of geometric form in modern furniture design. Travel Together. 14: 204.

2. Zhang Ruie, Liu Wenjia. (2011) Application of point, line and surface in furniture design. Industrial Science Tribune. 2:206-207.

3. Lv Jin. (2012) Discussion on the trend of modern furniture popular style. Modern Decoration (Theory). $1: 10$.

4. Liu Rui. (2017) Design expressiveness of furniture materials. Art and Literature of the Masses. 9:7879.

5. Wang jie, Li Ke. (2012) Analysis on semiotics of furniture design. Art and Literature of the Masses. 17:93-94.

the color matching is natural and lively, matching with its modeling, reflecting the feeling of fashion and personality. 